\title{
Déficit fiscal e inflación en El Salvador (1978-1987),
}

\author{
Alvaro Trigueros A.
}

Tradicionalmente los monetaristas le asignan un papel muy importante a la cantidad de dinero como determinante de los precios. Una de las principales razones que se mencionan como determinante de la cantidad de dinero ha sido el déficit fiscal. Con estos elementos afirman que un déficit fiscal financiado con préstamos del banco central aumenta directamente la cantidad de dinero, y esto a su vez genera un proceso inflacionario.

Lo característico de este planteamiento es que la causalidad de la relación va en una sola dirección, déficit fiscal-cantidad de dineroinflación. En esta investigación trataremos de demostrar, que en El Salvador, como pais subdesarrollado esto no es asi. Más bien sostendremos que estas variables se condicionan mutuamente a lo largo del tiempo. Así tenemos que la inflación es un fenómeno que influye positivamente sobre el déficit fiscal, por lo que la cantidad de dinero se verá afectada, incidiendo nuevamente sobre la inflación, y el proceso se volverá a repetir.

La razón por la cual sostenemos que la inflación incide sobre el déficit fiscal descansa en el hecho que mientras el gasto público es capaz de mantener el ritmo de crecimiento para defenderse de la inflación, los ingresos ticales no pueden, y por lo tanto el déficit del gobierno se incrementará.

Estas ideas serán ampliadas a continuación. En la primera parte haremos la prueba de causalidad entre las tasas de inflación y el crecimiento de la oferta de dinero, para aseguramos que ésta se da en ambas direcciones. En la segunda parte del trabajo desarrollaremos un modelo econométrico elaborado por Aghevli y Khan $(1978)^{2}$. En la 
tercera parte aplicaremos el modelo a El Salvador adaptándolo a las circunstancias, y presentamos el análisis de los resultados. Finalmente, expondremos nuestras conclusiones.

\section{PRUEBA DE CAUSALIDAD}

Con el objeto de examinar rigurosamente la relación entre las tasas de inflación y el crecimiento de la oferta de dinero, someteremos las variables a la prueba formal de causalidad que ha sido desarrollada por Pierce (1977) y Pierce y Haugh (1977) ${ }^{3}$. En este contexto la causalidad es interpretada como adelantos y retardos entre las variables. Una serie, digamos los precios, estará influyendo a la otra, el dinero, si se le adelanta en el tiempo. Si, por el contrario, los precios se rezagan consitentemente atrás del dinero, entonces el dinero influye en los precios. La doble causalidad o "feedback" es el reflejo de una de las series adelantándose, y al mismo tiempo rezagándose, en relación a la otra serie.

El procedimiento básico puede ser resumido brevemente. Primero colocamos cada una de las variables a través de un filtro para remover cualquier existencia de autocorrelación. Esto elimina el efecto que sobre una variable tienen sus propios valores pasados y la convierte en una serie con propiedades de ruido blanco ("white-noise": ésto es que los valores consecutivos de la serie no están autocorrelacionados). Para realizar esta pre-filtración utilizamos el filtro propuesto por Sims (1972) ${ }^{4}$ que consiste en transformar las series de la siguiente manera:

$$
\begin{array}{ll}
m_{1}=(1-0.75 L)^{2} & \log M_{1} \\
p_{1}=(1-0.75 L)^{2} & \log P_{1}
\end{array}
$$

Donde $m_{1}$ y $p_{1}$ son las series transformadas de el dinero y los precios respectivamente, $M_{1}$ y $P_{1}$ son las series originales de las mismas variables, y "L" es un operador de desfase, que al actuar sobre una variable la rezaga un periodo 5 .

Posteriormente obtenemos las correlaciones cruzadas de las dos series resultantes para ver si son independientes o si se relacionan. $\mathrm{Ya}$ que la correlación cruzada se ejecuta tanto con los valores rezagados como con los valores futuros de las series transformadas, esta prueba puede arrojar información sobre la dirección de la causalidad. Por ejemplo, un valor significativo del coeficiente de correlación entre el nivel de precios corriente y la oferta monetaria corriente, nos permite rechazar la hipótesis nula de que ambas series son independientes. Si sólo los valores rezagados de la tasa de crecimiento de la oferta de dinero son 
los que están-signitieativamente-corfolacionados-con los valores corrientes del nivel de precios, uno puede sostener que aumentos en lá cantidad de dinero influyen sobre los precios. Por otra parte, si sólo los valores futuros de la tasa de crecimiento del dinero son los que están significativamente correlacionados con los valores corrientes de la inflación, uno podría interpretar que la inflación influye sobre los aumentos de la oferta monetaria. Basados en este razonamiento, si ambas situaciones se cumplen simultáneamente implica que habrá una doble causalidad, en el sentido que el dinero influye sobre la inflación y viceversa.

\section{TABLA I}

El Salvador, primer trlmestre 1978 - cuarto trimestre 1987: correlaclón cruzada entre los preclos y el dinero. Con los preclos como varlable dependlente ${ }^{1}$

\begin{tabular}{ccc}
\hline Trimestre & $(-)^{2}$ & $(+)^{3}$ \\
\hline 0 & $0.9701^{*}$ & $0.9701^{*}$ \\
1 & $0.8651^{*}$ & $0.8439^{*}$ \\
2 & $0.8127^{*}$ & $0.8123^{*}$ \\
3 & $0.6796^{*}$ & $0.6979^{*}$ \\
4 & $0.6533^{*}$ & $0.6416^{*}$ \\
5 & $0.5566^{*}$ & $0.5057^{*}$ \\
6 & $0.4974^{*}$ & $0.4654^{*}$ \\
7 & $0.3862^{*}$ & $0.3685^{*}$ \\
8 & $0.3746^{*}$ & 0.3116 \\
\hline
\end{tabular}

1. El asterisco indica que el valor es mayor que el doble de su desviación típica, (1/ $\sqrt{N})$.

2. (-): indica la variable rezagada.

3. (+): indica la variable adelantada.

A continuación presentamos los resultados obtenidos de los coeficientes de correlación cruzada entre la tasa de inflación corriente y la tasa de crecimiento de la oferta de dinero de los ocho trimestres anteriores, de los ocho trimestres posteriores, y del mismo trimestre.

Los resultados nos indican que la inflación corriente está significativamente correlacionada con los valores pasados de la tasa de crecimiento de la cantidad de dinero hasta el octavo trimestre, y con los valores futuros de la misma hasta el séptimo trimestre, por lo que podemos concluir que existe una doble causalidad o "4eedback" entre las dos variables. 


\section{ESPECIFICACION DEL MODELO}

En esta sección presentamos el modelo econométrico que nos explica el mecanismo concreto a través del cual la inflación y la tasa de crecimiento de la cantidad de dinero son dos variables que se retroalimentan mutuamente.

\section{DETERMINANTES DE LOS PRECIOS}

Con el objeto de determinar los precios primero definimos la demanda de saldo monetarios ${ }^{6}$ reales como una función del ingreso real y del costo de oportunidad de mantener activos bajo la forma de dinero. En un país sub-desarrollado como El Salvador no existe una amplia gama de activos como alternativa al dinero, por lo que la sustitución entre dinero y activos físicos se hace más importante, de manera que el costo de oportunidad relevante es la tasa de retorno de los activos físicos o bienes duraderos, ésto es, la tasa de inflación esperada, mientras que la tasa de retorno de los activos financieros puede ser ignorada por el escaso desarrollo de este sector en el pais.

Por esto, especificamos la demanda de saldos monetarios reales en términos lineales aplicando logaritmos, de la siguiente manera:

$$
\log (M / P)^{d}=a_{0}+a_{1} \log Y_{1}-a_{2} \pi_{1} \quad a_{1}, a_{2}>0
$$

donde:

$$
\begin{aligned}
M= & \text { saldos monetarios nominales } \\
P= & \text { nivel de precios } \\
Y= & \text { nivel de ingreso real } \\
\pi= & \text { tasa de inflación esperada } \\
& \text { El índice "d" significa demanda. }
\end{aligned}
$$

La función ha sido expresada en términos logaritmicos esencialmente por razones de conveniencia, esto es, porque se pueden obtener directamente las elasticidades?.

Suponemos que los saldos monetarios reales actuales se ajustan proporcionalmente a la diferencia entre la demanda de saldos monetarios reales y los saldos existentes el periodo anterior,

$$
\Delta \log (\mathrm{M} / \mathrm{P})_{\mathrm{t}}=\Gamma\left(\log (\mathrm{M} / \mathrm{P})_{\mathrm{t}}^{\mathrm{d}}-\log (\mathrm{M} / \mathrm{P})_{\mathrm{t}-1}\right)
$$

donde $\Gamma$ representa el coeficiente de ajuste, $1>\Gamma>0$. 
La tasa de inflación esperada es generada por expectativas adaptativas, el mecanismo de aprendizaje por prueba y error propuesto por Phillip Cagan en 1956,

$$
\Delta \pi_{t}=\beta\left(\Delta \log P_{1}-\pi_{t-1}\right)
$$

donde $\beta$ y $\Delta \log P$, representan el coeficiente de expectativas y la tasa de inflación corriente, respectivamente.

Sustituyendo la ecuación (1) en la (2) y resolviendo para el nivel de saldos monetarios reales, obtenemos:

$$
\log (M / P)_{1}=\Gamma a_{0}+\Gamma a_{1} \log Y_{1}-\Gamma a_{2} \pi_{1}+(1-\Gamma) \log (M / P)_{1-1}
$$

Ya que estamos interesados en el nivel de precios, podemos resolver para obtener:

$$
\log P_{1}=-\Gamma a_{0}-\Gamma a_{1} \log Y_{t}+\Gamma a_{2} \pi_{t}-(1-\Gamma) \log (M / P)_{t-1}+\log M_{1}
$$

\section{SECTOR PUBLICO}

En el modelo original Aghevli y Khan (1978) suponen que el gasto real deseado por el gobierno está relacionado con el nivel de ingreso real, sin embargo para el caso de El Salvador, durante el periodo 19781987 no seria razonable suponer esto, ya que la prueba econométrica no arrojó resultados significativos, probablemente por las distorsiones que el financiamiento de la guerra provoca en el gasto público.

Por esto consideramos apropiado pensar que el gasto público nominal deseado por el gobierno está asociado con el ingreso nominal,

$$
\log G_{1}^{d}=g_{0}+g_{1}\left(\log Y_{1}+\log P_{1}\right) \quad g_{1}>0
$$

Donde $G$ es el gasto público nominal, $y$ las otras variables se definen como antes. Esto equivale a suponer que en el largo plazo el gobierno desea incrementar su gasto proporcionalmente al crecimiento del ingreso nominal y por tanto, podriamos esperar que $g_{1}$, la elasticidad ingreso nominal del gasto público, sea igual a la unidad, sobre todo en el caso de El Salvador donde las necesidades del gasto público están sometidas al financiamiento de la guerra (aunque esto no lo imponemos como una restricción en la estimación del modelo).

El gasto nominal actual se especifica como un ajuste sobre la diferencia entre el gasto nominal deseado y el gasto nominal del periodo anterior , 


$$
\Delta \log G_{1}=\sigma\left(\log G_{1}^{d}-\log G_{1-1}\right)
$$

donde $\sigma$ es el coeficiente de ajuste, $1>\sigma>0$.

Sustituyendo la ecuación (5) en la ecuación (6), obtenemos una solución para el nivel de gasto nominal,

$$
\log G_{1}=\sigma g_{q}+\sigma g_{1}\left(\log Y_{1}+\log P_{1}\right)+(1-\sigma) \log G_{1-1}
$$

De esta ecuación, uno puede obtener el rezago medio o promedio en el tiempo, del ajuste en el gasto público nominal. Este rezago se define sencillamente como $(1-\sigma) / \sigma$.

El ingreso fiscal nominal deseado se supone que está funcionalmente relacionado don el ingreso nominal,

$$
\log R_{1}^{d}=t_{0}+t_{1}\left(\log Y_{1}+\log P_{1}\right) \quad t_{1}>0
$$

donde $R$ representa el ingreso público nominal. Esperamos que la elasticidad del ingreso público, $t_{1}$, sea positiva.

El ingreso público actual se ajusta a la diferencia entre el ingreso público deseado y el ingreso público del periodo anterior,

$$
\Delta \log \mathrm{R}_{1}=\gamma\left(\log \mathrm{R}_{1}^{\mathrm{d}}-\log \mathrm{R}_{\mathrm{t}-1}\right)
$$

donde $\gamma$ es el coeficiente de ajuste, $1>\gamma>0$.

Sustituyendo la ecuación (8) en la (9), obtenemos una ecuación para el ingreso público nominal,

$$
\log R_{t}=\gamma_{0}+\gamma_{1}\left(\log Y_{1}+\log P_{1}\right)+(1-\gamma) \log R_{t-1}
$$

Si en el largo plazo el ingreso fiscal crece a la misma tasa que el ingreso nominal, podriamos esperar que $t_{1}$ sea igual a la unidad (esto es que en el largo plazo, gasto público e ingreso público podrían aumentar en el mismo porcentaje). Debería observarse que, en este marco teórico, aún si comenzamos con un presupuesto equilibrado, conforme el ingreso nominal crece, observaremos un aumento divergente entre el gasto público y el ingreso fiscal si el primero se ajusta más rápidamente. Esto es, el déficit fiscal nominal será función del incremento en el nivel de precios, bajo la condición $\sigma>r$, aún cuando $t_{1}=g_{1}$.

Hay razones suficientes para esperar que en El Salvador el gasto público nominal se ajuste más rápido a los incrementos en el ingreso 
nominal provenientes de la inflación, que los ingresos fiscales. Esto porque el gobierno encuentra, durante este periodo, serias dificultades para reducir sus obligaciones en términos nominales, por las exigencias de los gastos en materia de guerra. Por otro lado el ingreso fiscal nominal se rezaga sustancialmente del incremento en los precios por la baja elasticidad ingreso nominal de los sistemas impositivos y los largos retrasos en la recolección de impuestos. sobre la renta", ingreso queconcierne a los pagos de impuesto de un período dado, normalmente se retrasan más en los países subdesarrollados. En cualquier caso, los sistemas impositivos en estos países dependen fundamentalmente de los impuestos indirectos y en particular, de los impuestos al comercio exterior, para el caso de El Salvador la exportación se basa en productos agrícolas tradicionales, café, caña de azúcar y algodón. La notablemente mayor progresividad de los impuestos indirectos comparados con los directos es bien conocida. Además, los impuestos indirectos en los países en desarrollo son frecuentemente especificos, y aún cuando son ad-valorem, el ajuste de los valores de la base impositiva para algunos de estos impuestos no es suficientemente frecuente para mantener el paso con la inflación. Por ejemplo, el ajuste del tipo de cambio a la inflación doméstica es lento, lo que tiene efectos adversos en la recolección de impuestos provenientes del comercio exterior, y del precio de algunos productos básicos que pueden ser controlados, como la gasolina y el tabaco. Finalmente ocurren largas demoras en el revalúo periódico necesario para el impuesto a la propiedad.

\section{OFERTA DE DINERO}

La oferta de dinero, $M$, resulta de multiplicar la cantidad de dinero de alto poder, $H$, por el multiplicador monetario $\mathrm{m}$,

$$
M=m_{1} H_{1}
$$

Mediante algunas operaciones algebraicas se puede demostrar que ${ }^{10}$,

$$
H_{1}=G_{1}-R_{1}+E_{1}
$$

donde $\mathrm{E}$ es el residuo que contiene el cambio en las reservas internacionales netas, cambios en el crédito del banco central al sector privado, el total de dinero de alto poder en el periodo anterior y el error implicito en la diferencia del crédito del banco central al sector público comparada con el déficit fiscal, $G-R$.

La identidad (12) supone que un délicit fiscal provocará un aumento 
igual en la cantidad de dinero de alto poder. Esto podria ser cierto bajo la condición que el déficit fiscal sea financiado a través de préstamos del banco central, o utilizando saldos en efectivo retenidos en el banco central, prestando del extranjero, o prestando en los bancos comerciales bajo la condición de que estos rellenen sus reservas recurriendo al banco central, o con donaciones de paises extranjeros. Por otro lado cuando los déficits son financiados con préstamos de los bancos comerciales sin el descuento al banco central y prestando al sector privado no bancario, este supuesto no será válido. En El Salvador el campo de acción del último tipo de préstamos queda restringido por el escaso desarrollo del mercado tinanciero, de manera que nuestro supuesto previo puede ser considerado como válido. La ecuación de la oferta de dinero puede ser escrita como:

$$
M_{1}=m_{1}\left(G_{1}-A_{1}+E_{1}\right)
$$

El uso de la identidad (13) con nuestras otras ecuaciones podria hacer a nuestro modelo no lineal en las variables; $y$ ya que, desde el punto de vista de la estimación es más conveniente trabajar con modelos lineales, aproximaremos la ecuación (13) a una relación lineal en logaritmos, que fue obtenida linealizando respecto a medias muestrales.

El resultado de la ecuación lineal es:

$$
\log M_{1}=\log m_{1}+k_{0}+k_{1} \log G_{1}-k_{2} \log R_{1}+k_{3} \log E_{1}
$$

donde los parámetros $k_{0}, k_{1}, k_{2}$ y $k_{3}$ son funciones de las medias muestrales de $\log G, \log R$ y $\log E$. Los valores de los parámetros en la ecuación (14) pueden obtenerse calculando las medias muestrales de los logaritmos de las variables $G, R$, y E y derivándolos después, o estimándolos en el modelo junto con los otros parámetros.

\section{EL MODELO COMPLETO}

El modelo completo se resume en la tabla siguiente. El proceso inflacionario inherente a este sistema de ecuaciones puede ser resumido brevemente. Supongamos inicialmente que hay un incremento en la oferta de dinero a través de un incremento en "E" en la ecuación (18). Esto incrementaria el nivel de precios de la ecuación (15), que resultaría en aumentos tanto en el gasto público, ecuación (16), como en el ingreso fiscal, ecuación (17), y también en la inflación esperada, ecuación (19). Si el aumento en el gasto público es mayor que el del 
ingreso fiscal, el déficit fiscal se incrementará. Esto causará un incremento adicional en la oferta de dinero y el proceso se repetirá.

\section{ESTIMACION DEL MODELO Y ANALISIS DE LOS RE- SULTADOS}

A continuación procedemos a la estimación del modelo aplicándolo a El Salvador durante el periodo 1978-1987, para to cual utilizamos datos trimestrales (ver Apéndice III). Dado que es un modelo simultáneo, un método de estimación adecuado es el de mínimos cuadrados en dos etapas, de esta manera se garantiza que los parámetros estimados tengan varianza minima y que sean insesgados. Los resultados estimados se presentan en la tabla III.

TABLA II

Ecuaciones estructurales

$$
\begin{aligned}
\log P_{1} & =-\Gamma a_{0}-\Gamma a_{1} \log Y_{1}+\Gamma a_{2} \pi_{1}-(1-\Gamma) \log (M / P)_{t-1}+\log M_{1} \\
\log G_{1} & =\sigma g_{0}+\sigma g_{1}\left(\log Y_{1}+\log P_{1}\right)+(1-\sigma) \log R_{t-1} \\
\log R_{1} & =\gamma_{0}-\gamma_{1}\left(\log Y_{1}+\log P_{1}\right)+(1-\gamma) \log G_{1-1} \\
\log M_{1} & =\log m_{1}+k_{0}+k_{1} \log G_{1}-k_{2} \log R_{1}+k_{3} \log E_{1} \\
\pi_{1} & =\beta \Delta \log P_{1}+(1-\beta) \pi_{1-1}
\end{aligned}
$$

\section{variables endógenas}

$P$ : nivel de precios

G: gasto püblico nominal

$\mathrm{R}$ : ingresos fiscales nominales

$M$ : cantidad de dinero

$\pi$ : tasa de inflación esperada

\section{variables exógenas:}
Y: nivel de ingreso real
m: multiplicador monetario
E: variable residual

En una primera aproximación a los resultados, obervamos que en las ecuaciones 1, 4 y 5 casi todos los parámetros son estadisticamente significativos al $1 \%$, y lo mismo vale para los signos esperados, el coeficiente de determinación ajustado $\left(\mathrm{R}^{2}\right)$ y el estadístico $F$ sugieren que están correctamente especificadas y de acuerdo al criterio de la prueba " $h$ " o de la prueba durbin-watson (D.W.), según el caso, ninguna de dichas ecuaciones presentó el problema de autocorrelación, salvo la 
TABLA III

Ecuaciones estructurales estimadas'

1978 (primertrimestre)-1987 (cuartotrimestre) $\begin{aligned} \text { 1. } \log P_{1}= & \begin{array}{l}4.669-0.859 \\ (4.570)^{*}\end{array}(5,192)^{*} Y_{1}+2.289 \pi_{1}-0.276 \log (M / P)_{1-1} \\ (3.076)^{*} & (2.642)^{*}\end{aligned}$ $\ldots+\log M_{t}$

$R^{2}=0.735 ; \quad$ D.W. $=2.233 ; \quad h=-0.983 ; \quad F=37.020$

2. $\log G_{1}=-7.403+1.090\left(\log Y_{1}+\log P_{1}\right)-0.104 \log G_{1-1}$ $(3.914)^{*} \quad(4.719)^{*} \quad(0.505)$

$R^{2}=0.748 ; \quad$ D.W. $=1.914 ; \quad h=$ n.e.; $\quad F=58.924$

3. $\log R_{1}=-5.337+0.896\left(\log Y_{1}+\log P_{1}\right)-0.031 \log R_{1-1}$ $\begin{array}{lll}(3.534)^{*} \quad(5.174)^{*} & (0.187)\end{array}$

$R^{2}=0.659 ; \quad$ D.W. $=2.240 ; \quad h=$ n.e.; $\quad F=38.657$

4. $\log M_{1}=\log m_{1}+\underset{(0.631)}{0.235}+\underset{(5.856)^{*}}{0.249} \log G_{1}-\underset{(2.644)^{*}}{0.207} \log R_{1}+\ldots$ $\ldots+0.935 \log \mathrm{E}_{1}$

$(8.174)^{*}$

$\mathrm{A}^{2}=0.970 ; \quad$ D.W. $=1.916 ; \quad F=313.267$

5. $\pi_{1}=0.175 \log P_{1}+0.795 \pi_{1-1}$ $(2.077)^{* *}$

(9.486)

$R^{2}=0.280 ; \quad$ D.W. $=2.102 ; \quad h=-0.382 ; \quad F=16.167$

1. ()$=$ estadlstico $t$. Un asterisco (") indica que ol parámetro es significativo al $1 \%$, dos asteriscos ("*) que lo es al $5 \%$.

cuarta ecuación que fue corregida estimándola mediante del método de Cochran-Orcutt. Por su parte las ecuaciones 2 y 3 referentes al sector público plantean dos problemas. El primero es que en ambas el parámetro correspondiente al coeficiente de ajuste no es estadisticamente siginificativo. $Y$ en segundo lugar, para ninguna de las dos ecuaciones se puede conocer si existe o no el problema de autorrelación, porque el estadistico " $h$ " no se puede calcular al presentarse con una ralz cuadrada negativa. Por tanto si queremos ser rigurosos en la inter- 
pretación que los resultados, esto nos obliga a replanter las ecuaciones que se refieren al comportamiento de dicho sector. En realidad se trata de una sencilla especificación tomando en cuenta los hechos. Supondremos simplemente que tanto el gasto público como los ingresos fiscales van a depender de el ingreso real y de la inflación a fin de conocer directamente las elasticidades de ambos rubros con respecto a nuestras variables,

$$
\begin{aligned}
& \log G_{1}=g_{0}+g_{1} \log Y_{1}+g_{2} \log P_{1} \\
& \log R_{1}=t_{0}+t_{1} \log Y_{1}+t_{2} \log P_{1}
\end{aligned}
$$

En todo caso estas ecuaciones nos reflejarán la capacidad de los ingresos fiscales y del gasto público para mantener su crecimiento con respecto a la inflación y al ingreso real. Aqui se espera que la mayor influencia esté dada por la inflación, por el hecho que en El Salvador durante el periodo 1978-1987 el crecimiento del ingreso real ha sido negativo algunos años, y los años en que ha sido positivo fue a niveles bastante bajos, mientras que los precios han ido creciendo permanentemente. De esta manera la dinámica explicativa del modelo anterior sigue siendo prácticamente la misma, con la reserva de las modificaciones realizadas.

Aplicando nuevamente el método de los mínimos cuadrados en dos etapas, estimamos el modelo con la modificación precedente.

Procedemos ahora al análisis de los resultados de la tabla IV. Vale aclarar que las ecuaciones 2 y 4 de dicha tabla presentaron el problema de la autocorrelación, y que por tanto en la segunda etapa de la estimación se utilizó el método de Cochra-Orcutt.

Con respecto a la ecuación de los precios el ajuste es bastante alto lo mismo que el estadístico $F$, a su vez la prueba $h$ indica que no hay correlación serial. Sin embargo el parámetro que corresponde al término (1- $\Gamma)$ no tiene el signo correcto, y tampoco es estadísticamente significativo, por lo que no podemos tener ningún indicador que nos revele la velocidad de ajuste de la demanda de dinero y su efecto sobre los precios. A pesar de esta inconveniente, el resto de parámetros son estadisticamente significativos al nivel del $1 \%$, por lo que podemos afirmar que las elasticidades ingreso e inflación esperada de la demanda de dinero sí arrojan información consistente. Asi tenemos que la elasticidad ingreso de la demanda de dinero es igual a 0.8 , y la elasticidad inflación esperada de la demanda de dinero es igual a 8.5. Esto nos dice que al aumentar el ingreso en $1 \%$ la demanda de dinero aumentará en $0.8 \%$, lo que reducirá la presión sobre la inflación. 
Mientras que al aumentar las expectativas inflacionarias en $1 \%$, la demanda de saldos monetarios disminuye en aproximadamente $8.5 \%$, por lo que la presión sobre la inflación aumenta considerablemente, sugiriendo asi la importancia que tienen las expectativas de los salvadorenos en el proceso inflacionario del pais. Por otro lado la ecuación es suficientemente significativa en su conjunto como para poder afirmar que la última variable a la derecha, esto es, la cantidad de dinero, tiene una incidencia importante en el país.

\section{TABLAIV}

Ecuaclones estructurales estimadas' 1978 (prlmer trlmestre) - 1987 (cuarto trimestre)

1. $\log P_{t}=3.823-0.836 \log Y_{t}+8.902 \pi_{t}+0.040 \log (M / P)_{t-1} \ldots$ $\begin{array}{llll}(5.556)^{*} \quad(7.632)^{*} & (7.517)^{*} \quad(0.472)\end{array}$ $\ldots+\log M_{1}$
$R^{2}=0.878$;
D.W. = 2.109;
$h=-0.409$
$F=94.981$

2. $\log \mathrm{G}_{\mathrm{t}}=3.677-0.441 \log \mathrm{Y}_{1}+0.844 \log \mathrm{P}_{1}$ (1.665) (1.491) $(14.217)^{*}$

$$
\mathrm{R}^{2}=0.849 ; \quad \text { D.W. }=2.115 ; \quad F=73.974
$$

3. $\log \mathrm{R}_{\mathrm{t}}=-3.382+0.639 \log \mathrm{Y}_{\mathrm{t}}+0.827 \log \mathrm{P}_{\mathrm{t}}$
$(0.977)$
(1.375)
$(8.187)^{*}$

$$
R^{2}=0.667 ; \quad \text { D.W. }=2.271 ; \quad F=39.981
$$

4. $\log M_{t}=\log m_{1}+0.165+0.277 \log G_{1}-0.275 \log A_{1}+\ldots$
(0.354)
$(6.252)^{*}$
$(2.472)^{*}$ $\ldots+0.976 \log \mathrm{E}_{\mathrm{t}}$ $(6.515)^{*}$

$$
\mathrm{R}^{2}=0.972 ; \quad \text { D.W. }=1.823 ; \quad F=338.262
$$

5. $\pi_{t}=0.132 \log P_{t}+0.811 \pi_{1-1}$ $(2.004)^{\star *}$

$(10.110)^{*}$
$R^{2}=0.275$
D.W. = 2.108;
$h=-0.397$
$F=15.788$

1. ( ) = estadístico t. Un asterisco (") indica que el parámetro es significativo al $2 \%$ por lo menos, dos asteriscos ("*) que lo es al $5 \%$. 
Refiriéndonos a las ecuaciones que corresponden al sector público, ambas tienen el coeficiente de determinación ajustado $\left(\mathrm{R}^{2}\right)$ relativamente alto, $y$ el estadistico $F$ indica que las ecuaciones en su conjunto son estadísticamente significativas, así como el problema de correlación serial ya fue debidamente ajustado en la ecuación 2, mientras que la tercera ecuación no presentó problemas en este sentido. Las elasticidades ingreso real del gasto público nominal y de los ingresos fiscales nominales no son estadisticamente significativas, lo que tiene sentido si tomamos en cuenta lo que se advirtió en la especificación de las ecuaciones sobre el comportamiento del ingreso real en estos diez anos. En cambio las elasticidades precio si resultaron significativas al nivel del $1 \%$; dado que $0.844>0.827$, esto es que $g_{1}>t_{1}$, podemos afirmar que el gasto público nominal responde mejor al crecimiento de los precios que los ingresos fiscales, lo cual confirma nuestro argumento de que el proceso inflacionario tiende a presionar para que el déficit fiscal nominal se expanda.

La cuarta ecuación muestra un ajuste bastante elevado, y el estadístico $F$ nos indica que es altamente significativa. Lo mismo sus coeficientes son siginificativos al $2 \%$, y todos sus signos corresponden a los esperados, por tanto podemos concluir que la existencia de un déficit fiscal nos traerá como consecuencia un incremento de la oferta monetaria.

Finalmente la ecuación de expectativas racionales tiene un coeficiente de determinación bajo, pero el estadístico $F$ sugiere que en su conjunto es estadisticamente significativa. Sus dos coeficientes son significativos, al $5 \%$ el primero y al $1 \%$ el segundo, y tienen los signos esperados; y como también era de esperarse, la suma de ambos es cercana a uno, $0.132+0.812=0.944 ;$ podemos concluir de esto, que los salvadoreños han ajustado sus expectativas lentamente, a razón de $13.1 \%$ por trimestre, 10 que de alguna manera afecta la inflación, como se vió en la primera ecuación.

Observando el modelo en su conjunto, podemos asegurar que se prueba nuestra hipótesis inicial sobre la doble causalidad que existe entre la tasa de inflación y el crecimiento de la cantidad de dinero, y que el mecanismo de trasmisión de este fenómeno es el déficit fiscal que se financia con mayor emisión de moneda, fundamentalmente porque los gastos públicos crecen más rápido que los ingresos, en respuesta a la inflación.

\section{CONCLUSIONES}

A partir de los resultados anteriores podemos sacar algunas conclu- 
siones sobre el comportamiento de estas variables en nuestro país.

En primer lugar se observa, que en efecto, en nuestro pais el crecimiento del déficit fiscal en los últimos años sí afecta el proceso inflacionario al exigir una mayor emisión de dinero a las autoridades monetarias. $Y$ por otro lado el déficit fiscal tiende a crecer más a medida que los precios suben. Sin embargo, al momento de entrar en materia de política económica, no podemos ir más alla de sugerir que la reducción del déficit fiscal juega un papel importante en cualquier política anti-inflacionaria que se desee llevar a cabo, pero vale hacer la advertencia de que no es el único factor que incide sobre el proceso inflacionario, ya que hay otros elementos que en esta investigación no han sido tocados y que sin embargo son muy importantes". Por otro lado, una reflexión similar podemos hacer para el crecimiento del déficit fiscal, ya que si bien el modelo ha demostrado que la inflación tiende a incrementarlo, hay otros elementos que son determinantes del déficit fiscal, entre varios factores podemos mencionar los gastos de la guerra y la débil elasticidad tributaria del país.

Finalmente se concluye que no se pueden hacer sugerencias simplistas, como aquellas que aseguran que la inflación es solamente un fenómeno monetario y que para combatirla basta con reducir el déficit fiscal, pues la complejidad del problema demanda más estudios y análisis serios que se ubiquen a la altura de los grandes desequilibrios macroeconómicos que están llamados a resolver.

\section{NOTAS}

1. El presente ensayo es el resultado de una investigación realizada bajo la orientación de Roberto Rivera C. en la cátedra de ECONOMETRIA II.

2. Aghevli, Bijan B. y Mohsin S. Khan. "Government Deficits and the Inflationary Process in Developing Countries". IMF Staff Papers. Vol. 25 (september 1978), pp. 383-416.

3. Pierce, D.A., "Relationships - and the Lack There of-between Economic Time Series, with Special Reference to Money and Interest Rates". Journal of the American Satistlcal Assoclatlon, Vol. 72 (March 1977), pp. 1122.

Pierce, and L. D. Haugh, "Causality in Temporal Systems: Characterizations and a Survey". Journal of Econometrics, Vol. 5 (March 1977), pp. 265. 293.

Citados por Aghevli y Khan (1977), Ibid., pp. 386.

4. Sims. Christopher A., "Money, Income, and Causality", American Economic Revlew, Vol. LXII, № 4 (1972), pp. 545.

5. Los resultados se presentan en el Apéndice $\mathrm{I}$.

6. Se entiende por saldos monetarios lo que tradicionalmente se conoce como 
M2, esto es la suma de efectivo en poder del público, los depósitos a la vista del sector privado, depósitos a plazo, de ahorro y en divisas de residentes.

7. Sin embargo con respecto a la tasa de inflación esperada nuestra ecuación es realmente semilogarítmica.

8. En econometría esto se conoce como "hipótesis de ajuste parcial". Cir. Wallis y Stewart (1984), pp. 41-43 y Gujarati (1985), pp. 281.

9. Este punto es sumamente importante por la pérdida de valor real que sufren los ingresos fiscales cuando existe un procesu inflacionario durante este período de rezago. Cfr. Tanzi (1977).

10. Ver el apéndice II.

11. Un excelente estudio sobre el proceso inflacionario en El Salvador se encuentra en Roberto Rivera C. (1988). 
APENDICE I

Cantidad te dinero $(m)$ ypreclos $(p)$

variables transformadas

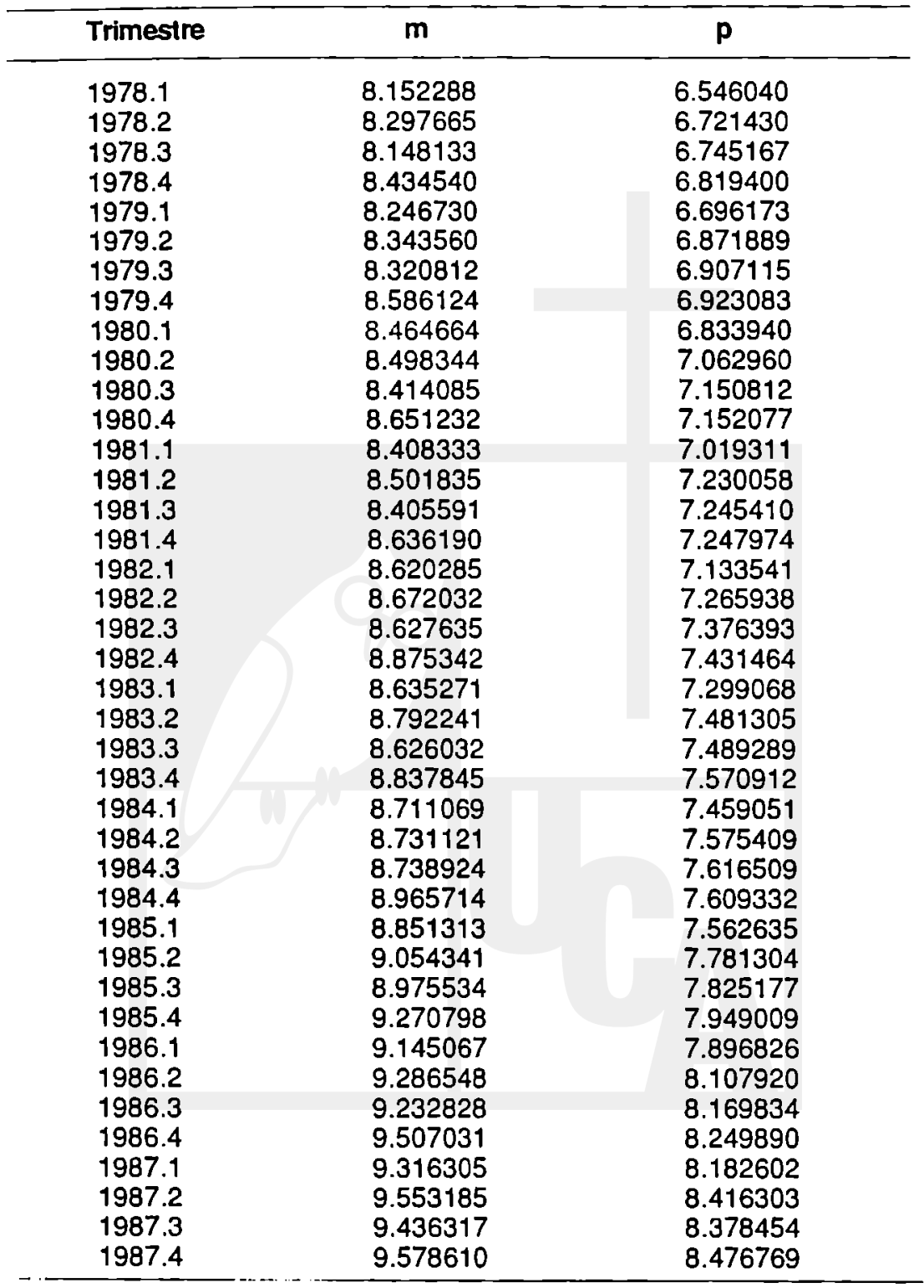




\section{APENDICE II \\ Demostraclón de la Identldad 12}

A continuación procedemos a hacer la demostración para obtener la identidad 12.

Los cambios en la masa monetaria $(H)$ pueden ocurrir a través de cambios en las reservas internacionales, cambios en el crédito del Banco Central al gobierno $(\Delta \mathrm{CG})$ y cambios en el crédito del Banco Central a los bancos comerciales y al sector privado. A su vez, los cambios en las reservas internacionales son el resultado de cambios en la balanza comercial y de capitales, créditos y donaciones provenientes del exterior, más otros flujos que incluye la balanza de pagos. Si consolidamos los cambios en las reservas internacionales y los cambios en el crédito al sector privado en una sola variable compuesta $(\triangle O A)$, podemos escribir

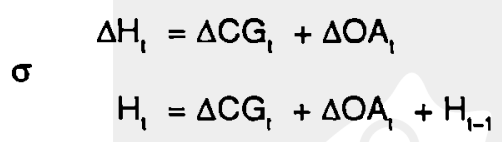

Por otra parte, si bien el déficit fiscal es financiado por el crédito del Banco Central al gobierno, este también puede ser financiado y de hecho lo es en El Salvador, por créditos y donaciones provenientes del extranjero, asi püdemos representar el déficit fiscal como:

$$
G_{1}-R_{1}=\Delta C G_{1}+\Delta C X t+\Delta D_{1}
$$

Esta ecuación nos refleja las fuentes de financiamiento del déficit fiscal, donde $C X_{t}$ corresponde al crédito del extranjero al gobierno, $D_{t}$ las donaciones del extranjero al gobierno, y las demás variables se definen como antes.

Resolviendo para $\triangle C G_{1}$ de la ecuación (2) y sustituyendo en (1') obtenemos,

$$
H_{1}=G_{1}-R_{1}+E_{1}
$$

donde $E_{t}=\Delta O A_{1}-\Delta C X_{t}+\Delta D_{1}+H_{t-1}$. Un aumento en el déficit fiscal se supone por tanto que provocará un aumento igual en la cantidad de dinero de alto poder. La identidad (3) arriba, es la que en el texto equivale a la identidad (12), esto es lo que queriamos demostrar. 
APENDICE III

Series utillzadas

(en mlllones de colones)

\begin{tabular}{|c|c|c|c|c|c|c|c|c|}
\hline & H & M & MUL & $\mathbf{P}$ & $\mathbf{R}$ & G & $Y$ & E \\
\hline 7.1 & 1077.500 & 400 & 009 & 358 & 900 & 500 & NA & 40 \\
\hline 7.2 & & & & & & & & \\
\hline 1977.3 & 933.7000 & 900.4000 & & 204.2296 & 280.9200 & 149.6500 & NA & 1064.970 \\
\hline 1977.4 & 1047.400 & 1099.800 & 1.050029 & 207.8550 & 183.9000 & 199.5300 & & 1031.770 \\
\hline 1978.1 & & & & & & & & \\
\hline 1978.2 & 1027.500 & 1059.400 & 1.031046 & 219.0332 & 338.5300 & 243.2600 & 869.5300 & 1122.770 \\
\hline 1978.3 & 858.6000 & 943.7000 & 1.099115 & 232.0242 & 217.1100 & 185.2200 & 0900 & 890.4900 \\
\hline 1978.4 & & & 1.1 & 22 & & & & \\
\hline 1979.1 & 000 & .600 & 1.15 & 244.7130 & 237.1000 & 152.8100 & 900 & 1046.990 \\
\hline 1979.2 & 000 & 109 & 1.1 & & 398.8300 & 197.1300 & 848.4600 & 1152.400 \\
\hline & 00 & & 1.1 & & 00 & & & \\
\hline 1979.4 & & & & & & 100 & & 310 \\
\hline & 12 & & 1.1 & & & 197. & & 460 \\
\hline 1980.2 & 000 & & 1.0 & & 00 & 22 & & \\
\hline & & & & & & & & \\
\hline 1980.4 & 132 & & $1 n$ & & & 360 & 100 & \\
\hline 1981.1 & 12 & & 1.0 & & 215 & 208.3800 & 834.4600 & in \\
\hline & & & & & & 279. & & \\
\hline & 10 & 11 & 1.0 & 22 & 000 & 323.7600 & & 300 \\
\hline & & & & & & 373 & & \\
\hline & 700 & 16 & 1.2 & & $\infty$ & 20 & & \\
\hline 198 & 200 & & & & 00 & 303.4500 & 300 & \\
\hline 19 & & & & & & 343. & & \\
\hline 1982.4 & 00 & & & & & 494. & & 580 \\
\hline 10 & & & & & 100 & 303. & & 180 \\
\hline 1983.2 & & & & & & & 00 & 620 \\
\hline & & & & & & & & \\
\hline & 152 & & & & & 475. & 716 & 750 \\
\hline & & & & & & & 00 & \\
\hline & & & & & & 359 & & \\
\hline 198 & 137 & & & & & 474 & 00 & \\
\hline & & 196 & 1.1 & & & & & \\
\hline & & & & & & & & \\
\hline & $1774 . \mathrm{con}$ & 0 & & 57 & 00 & 520 & 729. & \\
\hline & & & & & & & & \\
\hline & & & & & 528 & & 00 & \\
\hline & 2360.000 & 2480.000 & & 711.7825 & 483.7800 & 505.4900 & $785.060 \mathrm{C}$ & 23.38 .290 \\
\hline & 2379.000 & 2488.000 & & 765.5589 & 1136.190 & & & 2869.070 \\
\hline & & & & & & 100 & & \\
\hline & 2137.000 & 3047.000 & 1.42 & & 719.1400 & 680.6300 & 761.9000 & 2175.510 \\
\hline 1987.1 & & & & & & & & $23: 9.280$ \\
\hline & & & & & & $645.7 C$ & 754.2700 & \\
\hline & 25 & 2895.000 & 1.146354 & 1005.136 & 555.3300 & 705.2700 & 734.42 .00 & 2371.060 \\
\hline & 2675.000 & 147.000 & 1.176449 & 1045.619 & 825.7400 & 681.4800 & 800.0200 & 2819.260 \\
\hline
\end{tabular}

Fuentes: 1. Fondo Monetario Internaclonal, Estad/sticas Financieras Internacionales.

$\begin{array}{llll}H: & \text { bese monetaria; } & G: \text { Gasto público } \\ M: \text { cantidad de dinero, } M 2 ; & m: \text { multiplicador monelario }(m=M H) \\ P: \text { precios; } & E: \text { variable residual }(E=H-G+R)\end{array}$

A : ingresos fiscales;

2. Banco Central de Reserva

Y : Producto Interno Bruto a precios constantes de 1982. 


\section{BIBLIOGRAFIA}

1. Aghevli, Bijan B. and Mohsin S. Khan (1978). "Government Deficlts and the Inflatlonary Process in Developing Countrles". IMF Staff Papers, September.

2. 'Aghevli, Bijan B. and Mohsin S. Khan (1977). "Inflatlonary Finance and the Dynamics of Inflation: Indonesla, 1951-1972". American Economic Review, June.

3. Gujarati, D. (1985). "Econometrla Básica". Mc Graw Hill

4. Heller, p. S. (1980). "Impact of Inflation on Flscal Pollcy In Developing Countrles". IMF Staff Papers, December.

5. Rivera, R. (1988). "La Inflaclón en El Salvador". Realldad económicosoclal, enero-febrero.

6. Sims, C. A. (1972). "Money, Income, and Causality". American Economic Review, September.

7. Tanzi, V. (1977). "Inflatlon, Lags in Collection, and the Real Value of Tax Revenue". IMF Staft Papers, March.

8. Wallis, K. F. y M. B. Stewart (1984). "Introducclón a la econometrla". Alianza Universidad. 